\title{
Fauna Edáfica Auxiliando a Recuperação de Áreas Degradadas do Córrego Brejaúba, MG
}

\author{
Natallia Maria de Freitas Vicente ${ }^{1}$, Juliana Novelli Curtinhas ${ }^{1}$, \\ André Lage Perez ${ }^{2}$, Lusinério Prezotti ${ }^{3}$ \\ ${ }^{1}$ Departamento de Biologia Animal, Universidade Federal de Viçosa - UFV \\ ${ }^{2}$ Unidade Regional Zona da Mata, Empresa de Pesquisa Agropecuária de Minas Gerais - EPAMIG \\ ${ }^{3}$ Instituto Metodista Izabela Hendrix
}

\begin{abstract}
RESUMO
Objetivou-se analisar a abundância da fauna edáfica de diferentes hábitats. Em uma área de mata secundária e outra em avançado estágio de degradação da região do Córrego Brejaúba, MG, foi coletada serapilheira e solo em pontos quadrantes sorteados. Invertebrados foram extraídos por triagem e funis de Berlesse. A abundância e riqueza foram maiores no ambiente de mata. No ambiente degradado, a ausência de cobertura vegetal provavelmente resultou na amostragem inferior da fauna edáfica. Colembolla, Oligochaeta e ácaros envolvidos nos processos fundamentais do compartimento solo-serapilheira auxiliam na recuperação de áreas degradadas por meio de estratégias que estimulem suas atividades.
\end{abstract}

Palavras-chave: bioindicadores, fauna edáfica, manejo sustentável.

\section{Soil Fauna Helping the Recovery of Degraded Areas in Corrego Brejauba, State of Minas Gerais, Brazil}

\begin{abstract}
Two areas were chosen in Corrego Brejauba, State of Minas Gerais in order to characterize soil and litter invertebrates: a secondary forest and an area in advanced stage of degradation. Invertebrates were extracted by sorting and Berlesse funnels. The abundance and richness were higher in the secondary forest. In the degraded environment, lack of vegetation cover probably resulted in smaller sampling of soil fauna. Key groups such as Colembolla, Oligochaeta and Mites, involved in the soil-litter fundamental processes, can help the recovery of degraded areas through strategies that encourage their activities.
\end{abstract}

Keywords: bio-indicators, soil fauna, sustainable management. 


\section{INTRODUÇÃO}

Como resultado de uma posição estratégica, o Estado de Minas Gerais possui um alto grau de biodiversidade. Entretanto, o processo de ocupação verificado no Estado, aliado a um histórico de atividades extrativistas, tem provocado uma crescente perda de sua diversidade biológica (Drummond et al., 2005). O Vale do Rio Doce e, notadamente, a microrregião do Médio Rio Doce são provavelmente as porções mais degradadas de Minas Gerais (Baruqui, 1982; Florisbelo, 2006). Atualmente a busca de medidas mitigadoras para a recuperação dessa região tornou-se urgente, visando restabelecer a produção, o melhoramento e recuperação da fertilidade dos solos e a biodiversidade.

O solo está intimamente relacionado com outro compartimento, a serapilheira, principalmente nas florestas tropicais. Nos sistemas onde os solos apresentam geralmente uma baixa fertilidade natural, a matéria orgânica vinda da serapilheira apresenta-se como principal fonte para o crescimento vegetal (Correia \& Oliveira, 2000). A infinidade de invertebrados e os microrganismos que habitam o solo mostram sensibilidade às práticas de manejo desse substrato (Correia, 2002; Nunes et al., 2009).

As principais atividades da fauna de solo são: regulação das populações de microrganismos e a desintegração dos resíduos vegetais para eventual digestão da microflora, sendo liberados, nesse processo, elementos nutrientes que fazem parte do complexo orgânico (Primavesi, 2002). Alguns desses elementos, como fósforo e nitrogênio, enquadram-se na categoria de macronutrientes do solo, necessários em grandes quantidades para o crescimento da vegetação (Odum, 1988).

Essa íntima relação entre o solo e sua fauna permite avaliar vários tipos de impacto, como, a poluição, manejo do solo (Baretta et al., 2006) e desmatamento. Segundo Primack \& Rodrigues (2001), a fauna edáfica é importante para a criação de estrutura do solo, enfatizando o restabelecimento de comunidades para a restauração de ambientes degradados. Esses invertebrados podem ser explorados nos processos de remediação e recuperação, pela sua participação nas transformações no ambiente, que resultam na criação de novos micro-hábitats e nichos, possibilitando o aumento da biodiversidade (Correia, 2002).

Tais características justificam o uso da fauna de solo como indicadora de modificações ambientais (Correia \& Oliveira, 2000). Em geral, a alteração da abundância, diversidade e composição do grupo de indicadores mede a perturbação do ambiente (Brown, 1997).

Este trabalho teve como objetivo analisar a abundância e riqueza da fauna de invertebrados de solo e de serapilheira de dois diferentes hábitats na comunidade do Córrego do Brejaúba, Minas Gerais, a fim de subsidiar a recuperação de áreas degradadas na região.

\section{MATERIAL E MÉTODOS}

\section{1. Área de estudos}

O projeto foi desenvolvido na Comunidade do Córrego do Brejaúba, que fica a aproximadamente $15 \mathrm{~km}$ de Governador Valadares, MG. A coleta de dados concentrou-se em duas áreas dessa região. Uma mata com vegetação secundária, localizada nas coordenadas $18^{\circ} 53^{\prime} 15^{\prime \prime}$ de latitude e $41^{\circ} 53^{\prime} 22^{\prime \prime}$ de longitude e de altitude $520 \mathrm{~m}$, com aproximadamente 4 ha. E outra área que se encontra em severo processo de degradação. Localizada nas coordenadas $18^{\circ} 54^{\prime} 13^{\prime \prime}$ de latitude e $41^{\circ} 53^{\prime} 15^{\prime \prime}$ de longitude e altitude $442 \mathrm{~m}$, o local foi usado pelos proprietários para pastagem e vem passando por queimadas desde as décadas de 70 e 80 . Sua extensão é de aproximadamente 6 ha e apresenta-se assolada por voçorocas.

As áreas inserem-se em uma das sete microbacias recentemente escolhidas pelo IEF que, através do projeto ITTO, pretende recuperá-las com mudas de vegetação nativa cultivadas pela própria comunidade.

\subsection{Extração e triagem dos invertebrados terrestres}

Foram realizadas três campanhas de coleta entre os meses de Setembro a Outubro de 2006, sendo obtidos 30 pontos amostrais no ambiente degradado e 18 na mata. 
Em cada ambiente, os pontos ao acaso foram demarcados com a ajuda de estacas, obedecendo à área previamente estabelecida de 50 x $50 \mathrm{~cm}$. Para garantir a independência entre as amostras, os pontos distavam, no mínimo, cinco metros um do outro. Primeiramente, foi retirado todo o resíduo vegetal (serapilheira) que estava dentro dos limites do quadrante, sempre no sentido margens - centro, evitando-se assim, uma maior evasão dos organismos. Nesse mesmo quadrante seguia-se uma escavação de $15 \mathrm{~cm}$ de profundidade no solo. Esse sedimento foi transferido para peneiras de malha fina, revirado e os animais encontrados foram colocados em frascos contendo $15 \mathrm{~mL}$ de solução álcool $70 \%$. Após a procura pelos invertebrados, o solo foi descartado.

Em laboratório, cada amostra de serapilheira foi triada, em câmara fechada. Os indivíduos encontrados foram transferidos para frascos. Após esse procedimento, o folhiço foi acondicionado em Funis de Berlesse. Sobre cada funil, foram instaladas lâmpadas de $60 \mathrm{w}$, que forneceram o calor necessário para o gradiente de umidade. $\mathrm{Na}$ base, foram colocados potes com $100 \mathrm{~mL}$ de solução fixadora álcool 70\%. Cada amostra de serapilheira foi mantida nessa condição durante 48 horas.

A solução dos potes, após esse intervalo, foi transferida para placas de petri e mediante a observação em lupa, os invertebrados de solo foram capturados com pincéis e reservados. Após as triagens, os animais foram separados de acordo com características morfológicas, montados e identificados.

\subsection{Análises estatísticas}

As análises estatísticas envolveram Modelagem Linear Generalizada, com respectiva análise de resíduos, objetivando verificar os efeitos da variável $\mathrm{x}$ ou explanatória (Tipo de Hábitat) sobre as variáveis e ou dependentes (Riqueza e abundância). Foi utilizada uma ANOVA, com distribuição de poisson, corrigida para sobredispersão quando necessário. As análises foram realizadas no software $\mathrm{R}$ version 2.7.1. Para a riqueza estimada foi utilizado jackknife de primeira ordem do programa EstimateS win 7 (Colwell, 2004). Os grupos formicidae e isoptera foram retirados para análise de abundância.

\section{RESULTADOS E DISCUSSÃO}

Foram coletados 5.006 indivíduos, sendo 4.991 destes encontrados no ambiente de mata em 27 táxons (Tabela 1). Os animais foram separados em grandes grupos taxonômicos. As vantagens desse tipo de técnica é, primeiramente, a facilidade de execução, uma vez que não é necessário o conhecimento profundo na taxonomia dos grupos e a construção de uma visão geral da biota, incluindo o conhecimento global dos organismos presentes

Tabela 1. Abundância dos grupos encontrados nos ambientes de mata e área degradada do Córrego do Brejaúba, Minas Gerais.

Table 1. Abundance of invertebrates groups found in forest areas and degraded areas of Córrego Brejaúba, Minas Gerais.

\begin{tabular}{|c|c|c|}
\hline Hábitat & Grupos & Abundância \\
\hline \multirow{26}{*}{ Mata } & Pseudoescorpiones & 15 \\
\hline & Aranae & 127 \\
\hline & Acari & 3709 \\
\hline & Oligochaetas & 23 \\
\hline & Diplura & 1 \\
\hline & Collembola & 709 \\
\hline & Isopoda & 9 \\
\hline & Diplopoda & 36 \\
\hline & Chilopoda & 91 \\
\hline & Blattodea & 62 \\
\hline & Larva-Coleoptera & 61 \\
\hline & Coleoptera & 5 \\
\hline & Larva - lepidoptera & 1 \\
\hline & Pupa - lepidoptera & 1 \\
\hline & Larva - Diptera & 18 \\
\hline & Psocoptera & 52 \\
\hline & Thysanoptera & 16 \\
\hline & Larva-Thysanoptera & 13 \\
\hline & Hemiptera & 28 \\
\hline & Sp 190 & 2 \\
\hline & Sp 75 & 1 \\
\hline & Orthoptera & 8 \\
\hline & Embioptera & 1 \\
\hline & Trichoptera & 2 \\
\hline & Dermaptera & 1 \\
\hline & Total & 4991 \\
\hline \multirow{6}{*}{ Degradada } & Aranae & 4 \\
\hline & Coleoptera & 1 \\
\hline & Coleoptera - Larva & 4 \\
\hline & Diptera & 3 \\
\hline & Hemiptera & 3 \\
\hline & Total & 15 \\
\hline
\end{tabular}


(Moço et al., 2005). Parece não haver sentindo a inaplicação das medidas de diversidade a outros grupos taxonômicos além de espécies, sendo bastante útil em comunidades conhecidamente diversas, como a fauna de solo (Correia \& Oliveira, 2000). As limitações para esse tipo de estudo podem ser contornadas avaliando-se os grupos em diferente ambientes, enfocando-se assim as alterações na estrutura da comunidade impostas por modificações ambientais (Moço et al., 2005).

O tipo de hábitat influencia na abundância $\left(\mathrm{x}_{1,46}^{2}=5029,5 ; \mathrm{p}<0,001\right.$, Figura 1) e riqueza $\left(\mathrm{x}_{1,46}^{2}=\right.$ 38.894; $\mathrm{p}<0,001$, Figura 2) da fauna de solo, tendo o ambiente de mata apresentado maior número de indivíduos no total de cada grupo e maior riqueza. Somente 15 indivíduos e seis grupos taxonômicos

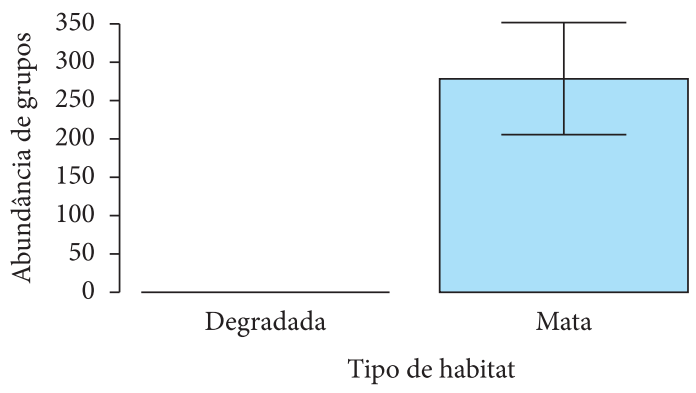

Figura 1. Relação de abundância dos grupos taxonômicos entre os dois hábitats. Demonstrando relação significativa na maior abundância no hábitat mata $\left(\mathrm{x}^{2}{ }_{1,46}=5029,5 ; \mathrm{p}<0,001\right)$.

Figure 1. Abundance ratio of taxonomic groups between habitats. Demonstrating significant relationship with greater abundance in the forest $\left(\mathrm{x}_{1,46}^{2}=5029,5 ; \mathrm{p}<\right.$ $0,001)$.

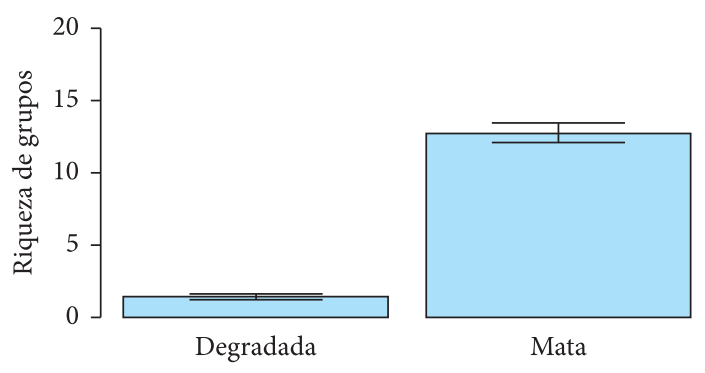

Tipo de habitat

Figura 2. Riqueza dos grupos nos diferentes hábitats. A mata apresentou maior diversidade de grupos $\left(\mathrm{x}_{1,46}^{2}=\right.$ $38.894, \mathrm{p}<0,001)$

Figure 2. Richness of taxonomic groups in different habitats. The forest showed the greatest diversity of groups $\left(\mathrm{X}_{1,46}^{2}=38.894, \mathrm{p}<0,001\right)$. foram encontrados no ambiente degradado, mostrando uma profunda perturbação nesse sistema. Hodkinson \& Jackson (2005) afirmam que a resposta de uma comunidade a perturbações é significante, pois reflete a mudança para múltiplas espécies, elevando juntamente o valor da perturbação.

Apesar de trabalhos ressaltarem o uso de formigas como indicadoras ambientais (Wink et al., 2005; Freitas et al., 2006), tratando-se de estudos de abundância, grupos de insetos sociais podem levar a uma análise errônea da realidade local. Em ambientes degradados, algumas espécies de formiga generalistas tendem a predominar nas amostras, uma vez que o efeito da modificação provavelmente eliminou os inimigos e competidores naturais.

A diferença de riqueza de espécies entre os hábitats, provavelmente, se deve às diferenças na complexidade da vegetação. Os resultados obtidos sugerem que a disponibilidade de micro-hábitats é o principal mecanismo de influência da vegetação sobre a fauna edáfica, já que existem vários estudos relacionando positivamente a complexibilidade do ambiente e a riqueza, uma vez que a complexidade do ambiente influencia na capacidade de suportar um grande número de espécies (Rego et al., 2003).

$\mathrm{Na}$ área de mata, a alta incidência de grupos pode ser explicada pela maior quantidade de serapilheira observada nesse hábitat. A adição de coberturas pode aumentar consideravelmente a infiltração, reduzir a evapotranspiração e a perda de matéria orgânica do solo (Wardle, 1995). A presença de serapilheira na mata, portanto, favoreceu a ocorrência de animais que necessitam da umidade para sua sobrevivência, como ácaros, colêmbolos, miriápodes, oligoquetos e crustáceos, que, segundo Lavelle (1997), fazem parte da mesofauna do solo. Esses animais realizam a fragmentação do material vegetal (Correia \& Oliveira, 2000), viabilizando novos micro-hábitats.

Blattodeas são reconhecidamente generalistas nos seus hábitos alimentares (Triplehorn \& Johnson, 2005) e apareceram somente no hábitat mata, provavelmente devido aos vários substratos, nichos, e recursos que esse ambiente ofereceu, o que não ocorre na área degradada.

A serapilheira foi esparsamente encontrada na área degradada; podendo ser efeito das práticas de queimadas da região. Nunes et al. (2009) observaram 
a diminuição da população edáfica em ambientes em que o uso do fogo era utilizado como manejo em sistemas de produção. A queimada de áreas para fins de plantio ou colheita tem efeitos negativos sobre a fauna de solo, pois elimina praticamente todos os animais, dificultando as relações ecológicas, reduz a fonte de alimento e abrigo (Correia \& Oliveira, 2000), induzindo o sucesso de sobrevivência para poucas espécies mais resistentes.

A alta amostragem de coleópteros e aranhas tanto no hábitat degradado quanto na mata ressaltam sua ampla distribuição e adaptabilidade. Nunes et al. (2008), em estudos na caatinga, ressaltam que os grupos formicidae e coleópteras apresentaram-se mais resistentes às condições adversas de manejo do solo nesse ambiente.

A ocorrência de ácaros somente no hábitat de mata, com mais de $50 \%$ da amostragem total, confirma a incidência desse grupo em ambientes equilibrados, com maior teor de matéria orgânica e umidade, recursos que acarretam o surgimento de fungos, dos quais muitos deles se alimentam (Ducatti, 2002). Isso também favoreceu, provavelmente, o aparecimento de outros grupos, como psocoptera, collembola e isopoda, que utilizam fungos e matéria orgânica como fontes de alimento (Triplehorn \& Johnson, 2005).

Táxons que apareceram no hábitat mata, como chilopoda, aranae, pseudoescorpioes, dermaptera, representam uma importante parte da teia trófica, os predadores. Esse grupo está intimamente relacionado com a estabilidade do sistema. A sua ausência pode levar a uma modificação na densidade de espécies (Merlim, 2005; Begon, 2006), o que provavelmente aconteceu no ambiente degradado, no qual a ausência de presas pode ter eliminado a ocorrência dos predadores.

Os oligoquetas não foram encontrados na área degradada. A compactação do solo pela pecuária, resultando no aumento de microporos e zonas anaeróbicas, pode ter causado a ausência desses. Esse grupo modifica profundamente a estrutura do solo através da escavação e seus coprólitos incorporam matéria orgânica aos perfis desse substrato (Andrade, 2000).

Sabendo-se que estágios de desenvolvimento dentro de uma mesma espécie ocupam diferentes nichos, influenciando a variedade do ecossistema (Odum, 1988), separaram-se as larvas de coleoptera e diptera para um melhor entendimento das interações. Em termos de funcionalidade, esses grupos podem ser saprófagos ou predadores (Moço et al., 2005). Larvas de coleóptera foram encontradas em menor quantidade na área degradada. A presença de fezes de animais (vacas, cavalos) pode estar atraindo coleopteras adultos de hábitos coprófagos em busca de alimento. Merlim et al. (2006), analisando a população de larvas de coleópteras em ecossistemas de Araucária, observaram uma maior riqueza de famílias em mata nativa em relação à mata introduzida, confirmando o potencial do uso desse grupo como indicadores ambientais.

A maior riqueza e abundância encontrada no hábitat mata em contraste ao degradado poderia ser reflexo de uma baixa amostragem. As curvas de acumulação observadas se mostraram estabilizadas a partir do $11^{\circ}$ ponto amostral para o hábitat degradado e uma tendência à estabilização a partir do $15^{\circ}$ ponto amostral no hábitat mata secundária. Apesar dessa tendência, a relação entre a riqueza observada e a estimada foi próxima $\left(\mathrm{S}_{\mathrm{obs}}=27\right.$, $\left.\mathrm{S}_{\text {esp }}=31,72\right)$, demonstrando que o esforço de coleta foi suficiente para caracterizar a comunidade edáfica das duas áreas, e reforçando assim o fato de que a diferença na fauna entre os hábitats é realmente causado pela diferença de estabilidade ambiental no hábitat degradado (Figura 3).

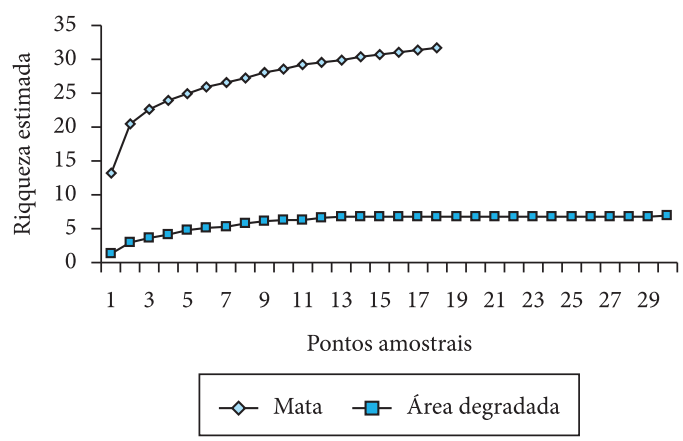

Figura 3. Curva de riqueza estimada (Jackniffe de primeira ordem) dos grupos taxonômicos nos dois hábitats, área degradada e mata do Córrego do Brejaúba, Minas Gerais.

Figure 3. Estimated richness curve (Jackniffe firstorder) of the taxa in the two habitats, forest and degraded areas in Córrego of Brejaúba, Minas Gerais, Brazil. 
As curvas estimadas tiveram desempenho diferente em cada tipo de hábitat, sendo reflexo da diferença significativa de riqueza entre eles.

Diante da associação entre grupos da fauna edáfica e sistemas de manejo do solo (Baretta et al., 2006; Nunes et al., 2009), é possível sugerir práticas agrícolas que prezem pela sustentabilidade. Santos et al. (2008) caracterizaram a macrofauna edáfica em sistema de plantio direto e encontraram efeito significativo das plantas de cobertura sobre os grupos taxonômicos e densidade relativa desses animais.

Estratégias que estimulem a atividade de invertebrado do solo podem auxiliar na remediação de áreas degradadas, como o hábitat degradado avaliado neste estudo, aumentando a fertilidade do solo desgastado por um histórico de pastejo e agricultura intensiva.

\section{CONCLUSÕES}

- Estratégias que visem a adição de coberturas e estabelecimento de umidade podem auxiliar na recuperação de áreas degradadas por estimularem atividades de grupos-chave, tais como Colembolla, Oligochaeta e ácaros, envolvidos nos processos fundamentais do compartimento solo-serapilheira.

- Ressalta-se relação das densidades de presapredador como importante ferramenta para medir o equilíbrio.

- A área degradada, que passará por um processo de recuperação, merece atenção, uma vez que o monitoramento pode revelar grupos pioneiros que indiquem a restauração do equilíbrio.

\section{STATUS DA SUBMISSÃO}

Recebido: 15/10/2009

\section{AUTOR(ES) PARA CORRESPONDÊNCIA}

\section{Natallia Maria de Freitas Vicente}

Departamento de Biologia Animal, Universidade Federal de Viçosa - UFV,

Av. Peter Henry Rolfs, s/n, Campus Universitário, CEP 36570-000, Viçosa, MG, Brasil e-mail: natalliavicentte@gmail.com

\section{REFERENNCIAS}

Andrade LB. O uso da fauna edáfica como bioindicadora de modificações ambientais em áreas degradadas [monografia]. Rio de Janeiro: Universidade Rural do Rio de Janeiro; 2000.

Baretta D, Mafra AL, Santos CP, Amarante CVT, Bertol I. Análise multivariada da fauna edáfica em diferentes sistemas de preparo e cultivo do solo. Pesquisa Agropecuária Brasileira 2006; 41: 1675-1679.

Baruqui FM. Inter-relações solo-pastagens nas regiões Mata e Rio Doce do Estado de Minas Gerais [dissertação]. Viçosa: Universidade Federal de Viçosa; 1982.

Begon M, Townsend CR, Harper JL. Ecology: from individuals to ecosystems. Blackwell Science, Oxford; 2006.

Brown KS. Diversity, disturbance, and sustainable use of Neotropical forests: insects as indicators for conservation monitoring. Journal of Insect Conservation 1997; 1: 25-42.

Colwell RK. Estimates: statistical estimation of species richness and shared species from sample. [cited 2009 abr. 10]. Version 7. 2004. Available from: http://purl. ocic.org/estimates.

Correia MEF, Oliveira LCM. Fauna de solo: aspectos gerais e metodológicos. [cited 2006 nov. 12]. Seropédica: Embrapa Agrobiologia. 2000. 46 p. Documentos, no. 112. Available from: http://www.cnpab.embrapa.br/ publicacoes/download/doc112.pdf.

Correia MEF. Relações entre a diversidade da fauna de solo e o processo de decomposição e seus reflexos sobre a estabilidade dos ecossistemas. [cited 2006 out. 28]. Seropédica: Embrapa Agrobiologia; 2002. Documentos, no. 156. Available from: http://www.cnpab.embrapa.br/ publicacoes/download/doc156.pdf.

Drummond GM, Martins CS, Machado ABM, Sebaio FA, Antonini Y. Biodiversidade em Minas Gerais: um Atlas para sua conservação. 2 ed. Belo Horizonte: Fundação Biodiversitas; 2005.

Ducatti F. Fauna edáfica em fragmentos florestais e em áreas reflorestadas com espécies da mata atlântica [dissertação]. Piracicaba: Escola Superior de Agricultura "Luiz de Queiroz"; 2002.

Florisbelo GR. Estudo propositivo para a dinamização econômica do território do Médio Rio Doce - MG. Brasília: Ministério do Desenvolvimento Agrário; 2006. $170 \mathrm{p}$.

Freitas AVL, Francini RB, Brown JR, Keith S. Insetos como indicadores ambientais. In Cullen Jr. L, Rudran R, Valladares-Padua, C. Métodos de estudos em biologia da conservação er manejo da vida silvestre. Curitiba: UFPR; 2004. p. 19-41. 
Hodkinson ID, Jackson JK. Terrestrial and aquatic invertebrates as bioindicators for environmental monitoring, with particular reference to mountain ecosystems. Environmental Management 2005; 35:649-666

Lavelle P. Faunal activities and soil processes: adaptative strategies that determinate ecosystem function. Advances in Ecological Research 1997; 27:93-132.

Merlim AO. Macrofauna edáfica em ecossistemas preservados e degradados de araucária no Parque Estadual de Campos do Jordão, SP [dissertação]. Piracicaba: Escola Superior de Agricultura "Luiz de Queiroz'; 2005.

Merlim AO, Aquino AM, Cardoso EJBN. Larvas de Coleoptera em ecossistemas de araucária no Parque Estadual de Campos do Jordão, SP. Ciência Rural 2006; 36:1303-1306

Moço MKS, Gama-Rodrigues EF, Gama-Rodrigues AC, Correia MEF. Caracterização da fauna edáfica em diferentes coberturas vegetais na região norte Fluminense. Revista Brasileira de Ciência do Solo 2005; 29:555-564.

Nunes LAPL, Araújo Filho JA, Menezes RÍQ. Diversidade da fauna edáfica em solos submetidos a diferentes sistemas de manejo no semi-árido nordestino. Scientia Agraria 2009; 10: 043-049.

Nunes LAPL, Araújo Filho JÁ, Menezes RÍQ. Recolonização da fauna edáfica em áreas de caatinga submetidas a queimadas. Caatinga 2008; 21:214-220.
Odum EP. Ecologia. Rio de Janeiro: Guanabara Koogan; 1988. 434 p.

Primack RB, Rodrigues E. Biologia da conservação. Londrina: Planta; 2001. 328 p.

Primavesi A. Manejo ecológico do solo: a agricultura em regiões tropicais. 7 ed. São Paulo: Nobel; 2002. 549 p.

Rego FNAA, Venticinque EM, Brescovit AD. Fragmentos florestais reduzem a abundância da comunidade de aranhas do sub-bosque, na Amazônia Central: Considerações sobre o estudo e a conservação de áreas degradadas. In Anais do IV Congresso de Ecologia do Brasil; 2003. 237 p.

Santos GG, Silveira PM, Marchão RL, Becquer T, Balbino LC. Macrofauna edáfica associada a plantas de cobertura em plantio direto em um Latossolo Vermelho do Cerrado. Pesquisa Agropecuária Brasileira 2008; 43:115-122.

Triplehorn CA, Johnson NF. Borror and Delong's introduction to the study of insects. $7^{\text {th }}$ ed. USA: Thomson-Brooks/Cole; 2005. 864 p.

Wardle DA. Impacts of disturbance on detritus food webs in agroecossistems of contrasting tillage a weed managements practices. Advances in Ecological Research 1995; 26:105-182.

Wink C, Guedes JVC, Fagundes CK, Rovedder AP. Insetos edáficos como indicadores da qualidade ambiental. Revista de Ciências Agroveterinárias 2005; $4: 60-71$ 